\title{
Decoherence and robustness of parity dependent entanglement in the dynamics of a trapped ion
}

\author{
S. Maniscalco, A. Messina, A. Napoli \\ INFM Unità di Palermo and Dipartimento di Scienze Fisiche ed Astronomiche \\ dell'Università di Palermo, via Archirafi 36, 90123 Palermo, Italy
}

\section{Vitali}

INFM Unità di Camerino and Dipartimento di Matematica e Fisica dell'Università di Camerino, via Madonna delle Carceri, 62032 Camerino, Italy

\begin{abstract}
We study the entanglement between the 2D vibrational motion and two ground state hyperfine levels of a trapped ion, Under particular conditions this entanglement depends on the parity of the total initial vibrational quanta. We study the robustness of this quantum coherence effect with respect to the presence of nondissipative sources of decoherence, and of an imperfect initial state preparation.

PACS numbers: 42.50.Dv, $42.50 . \mathrm{Vk}, 32.80 . \mathrm{Pj}$
\end{abstract}

\section{Introduction}

Over the last few years we have witnessed a very rapid development of cooling and trapping techniques both for neutral atoms and for ions [1, 2, 3, , 6, 5, 6, 7]. These progresses have made it possible to perform sophisticated experiments wherein several examples of couplings between a few bosonic and fermionic dynamical variables have been realised [8, [9, 9, 10, 11, 12]. If, in fact, an ion confined in a miniaturised Paul trap is exposed to suitably configured laser beams, the 3D harmonic motion of the ionic center of mass gets entangled with the internal degrees of freedom. Some peculiar aspects of the vibronic response stemming from such a dissipation-free situation have been successfully exploited for realizing experimentally Fock states, coherent states, squeeezed states and Schrödinger cat-like states 19, 10, 11], presenting theoretical schemes for engineering several nonclassical state 133, 14, 16, 15, 17, 18, 19, 20, 21, 22, 231, implementing quantum logic gates [8, 24, 25], realising tomographic reconstructions of the density matrix of the system [26, 27, 28, 29], and, more in general, discovering quantum effects characterising the ionic quantized oscillatory motion [30, 31, 32, 33, 34, 35]. Many hamiltonian models have been reported so far in the literature to describe physical properties of trapped ions. Some models explore physical situations wherein only the ionic motion along a specified direction of the trap is effectively influenced by the presence of the laser beams. When the induced vibronic coupling is instead extended over two (three) independent 
directions of the trap, then one refers to the correspondent physical scenario as to a $2 \mathrm{D}$ (3D) trapped ion.

In this paper we study the motion of an ion isotropically confined in the radial plane of a Paul microtrap when it is irradiated by a properly chosen configuration of external laser beams. We show that there exist experimentally interesting conditions under which the dynamics of this system may be exactly treated [22]. Exploiting this fact we find that the center of mass vibrational motion and the electronic degrees of freedom of the ion becomes entangled, in a way which is very sensitive to the parity of the initial number of vibrational quanta. Then we study the robustness of this quantum effect with respect to various experimental imperfections. In fact, even if dissipation of the vibrational motion is completely negligible, non-dissipative sources of decoherence associated with fluctuations of external parameters may be important. We study these effects, and those of laser intensity fluctuations in particular, using the model-independent formalism of Ref. [37]. We find that, using standard values for the strength of the laser intensity fluctuations, the parity-dependent entanglement effect is not washed out by non-dissipative decoherence. The effect of an imperfect initial state preparation of the vibrational degree of freedom is also studied.

\section{Parity dependent entanglement effect}

\subsection{Derivation of the effective Hamiltonian model}

Consider a two-level ion of mass $M$ confined in a bidimensional isotropic harmonic potential characterised by the trap frequency $\nu$. Let's denote by $\hbar \omega_{0}$ the energy separation between the ionic excited state $|+\rangle$ and its ground state $|-\rangle$ and assume $\omega_{0} \gg \nu$. Indicate by $a\left(a^{\dagger}\right)$ and $b\left(b^{\dagger}\right)$ the annihilation (creation) operators of vibrational quanta relative to the ionic center of mass oscillatory motion along the $x$ and $y$ axes of the radial plane of the trap respectively.

We assume that the ion is driven by two $\pi$-out of phase laser beams, applied along the two orthogonal directions $\bar{x}$ and $\bar{y}$ with an angle of $\pi / 4$ relative to the $x$ and $y$ axis respectively. The two lasers have equal frequency $\omega_{L}=\omega_{0}-2 \nu$, intensity $E_{0}$ and wavevector modulus $k_{L}$. After performing the dipole and the rotating wave approximations, the hamiltonian model of the system can be cast in the form

$$
\hat{H}=\hbar \nu\left(\hat{a}^{\dagger} \hat{a}+\hat{b}^{\dagger} \hat{b}\right)+\frac{\hbar \omega_{0}}{2} \hat{\sigma}_{z}+\left[d \epsilon^{(-)}(\bar{x}, \bar{y}, t) \hat{\sigma}_{-}+\text {h.c. }\right]
$$

where $d$ is the appropriate dipole matrix element, assumed real for simplicity, and $\epsilon^{(-)}(\bar{x}, \bar{y}, t)=E_{0}\left[e^{i\left(\omega_{L} t-k_{L} \bar{x}\right)}-e^{i\left(\omega_{L} t-k_{L} \bar{y}\right)}\right]$ represents the negative frequency contribution to the resultant electric field acting upon the atom. The two-level atomic degrees of freedom are described in terms of the inversion operator $\hat{\sigma}_{z}=|+\rangle\langle+|-|-\rangle\langle-|$ and of the two transition operators $\hat{\sigma}_{ \pm}=| \pm\rangle\langle\mp|$. In a reference frame rotating at the laser frequency $\omega_{L}$, the hamiltonian $\hat{H}$ assumes the form $\hat{H}_{L}=\hat{H}_{0}+\hat{H}_{1}$ with

$$
\hat{H}_{0}=\hbar \nu\left(\hat{a}^{\dagger} \hat{a}+\hat{b}^{\dagger} \hat{b}\right)+\frac{\hbar \delta}{2} \hat{\sigma}_{z}
$$


Decoherence and robustness...

$$
\hat{H}_{1}=\hbar \Omega\left\{\left[e^{-i \eta\left(\hat{A}+\hat{A}^{\dagger}\right)}-e^{-i \eta\left(\hat{B}+\hat{B}^{\dagger}\right)}\right] \hat{\sigma}_{-}+\text {h.c. }\right\}
$$

where

$$
\delta=\omega_{0}-\omega_{L}=2 \nu, \quad \Omega=\frac{d E_{0}}{\hbar}, \quad \eta=k_{L} \sqrt{\frac{\hbar}{2 \nu M}}
$$

are the atom-laser detuning, the Rabi frequency and the so-called Lamb-Dicke parameter respectively. We have indicated with $\hat{A}\left(\hat{A}^{\dagger}\right)$ and $\hat{B}\left(\hat{B}^{\dagger}\right)$ the annihilation (creation) operators of vibrational quanta along the directions $\bar{x}$ and $\bar{y}$ respectively, defined by

$$
\hat{A}=\frac{1}{\sqrt{2}}(\hat{a}+\hat{b}) \quad \hat{B}=\frac{1}{\sqrt{2}}(\hat{b}-\hat{a})
$$

In the interaction picture, the interaction Hamiltonian assumes the form

$$
\begin{aligned}
\hat{H}_{i n t} & =\hbar \Omega e^{-\eta^{2} / 2}\left\{\sum_{k, j=0}^{\infty}(-1)^{j+k} \frac{(i \eta)^{j+k}}{j ! k !} \hat{A}^{j}\left(\hat{A}^{\dagger}\right)^{k} e^{i \nu t(k-j-2)}+\right. \\
& \left.-\sum_{k, j=0}^{\infty}(-1)^{j+k} \frac{(i \eta)^{j+k}}{j ! k !} \hat{B}^{j}\left(\hat{B}^{\dagger}\right)^{k} e^{i \nu t(k-j-2)} \sigma_{-}+\text {h.c. }\right\}
\end{aligned}
$$

where the resonance condition $\delta=2 \nu$ has been used. Under the assumption $\nu \gg \Omega$, we may perform the rotating wave approximation neglecting in Eq. (6) all the terms oscillating at multiples of the harmonic trap frequency $\nu$. Finally, using Eqs. (5) it is not difficult to show that, in the Lamb-Dicke limit $\eta \ll 1$, the effective interaction Hamiltonian in the interaction picture can be written as [18, 20]

$$
\hat{H}_{i n t}=\hbar g\left(\hat{a} \hat{b} \hat{\sigma}_{+}+\text {h.c. }\right)
$$

with $g=\Omega \eta^{2} e^{-\eta^{2} / 2}$.

\subsection{Experimental set up}

For realizing the Hamiltonian model (7) we make use of a three level electronic system as shown in Fig. 1. Such a system is similar to the one currently used in the experiments performed at NIST [9, 10]. The weak transition $|-\rangle \leftrightarrow|+\rangle$ is simultaneously driven by two Raman laser configurations with different detunings $\left(\Delta_{1}\right.$ and $\left.\Delta_{2}\right)$ from the electronic level $|3\rangle$ and with frequency differences $\omega_{L}=\omega_{L_{1}}-\omega_{L_{2}} \equiv \omega_{L^{\prime}}=\omega_{L_{1}^{\prime}}-\omega_{L_{2}^{\prime}}=\omega_{0}-2 \nu$. For sufficiently large detunings, such that the intermediate state $|3\rangle$ can be adiabatically eliminated, only the electronic levels $|+\rangle$ and $|-\rangle$ are involved in the dynamics. In this case the two-photon Raman transitions between the states of interest are formally equivalent to narrow single photon transitions 99, 10]. The Raman laser fields are chosen to have wave vector differences $\vec{k}_{L}=\vec{k}_{L_{1}}-\vec{k}_{L_{2}}$ and $\vec{k}_{L^{\prime}}=\vec{k}_{L_{1}^{\prime}}-\vec{k}_{L_{2}^{\prime}}$ pointing in the $\bar{x}$ and $\bar{y}$ directions respectively. Under these conditions, in the resolved sideband regime and in the Lamb-Dicke limit, the dynamics of the system is described by the Hamiltonian (7) provided that we make the following substitution $g=\Omega_{R} \eta_{R}^{2} e^{-\eta_{R}^{2} / 2}$ with $\Omega_{R}=\Omega_{L_{1}} \Omega_{L_{2}} / \Delta_{1}=\Omega_{L_{1}^{\prime}} \Omega_{L_{2}^{\prime}} / \Delta_{2}$ and $\eta_{R}=\sqrt{\hbar k_{L}^{2} / 2 M \nu}=\sqrt{\hbar k_{L^{\prime}}^{2} / 2 M \nu}$. The dependence of the Lamb-Dicke parameter on the modulus of the wave vector difference allows to 


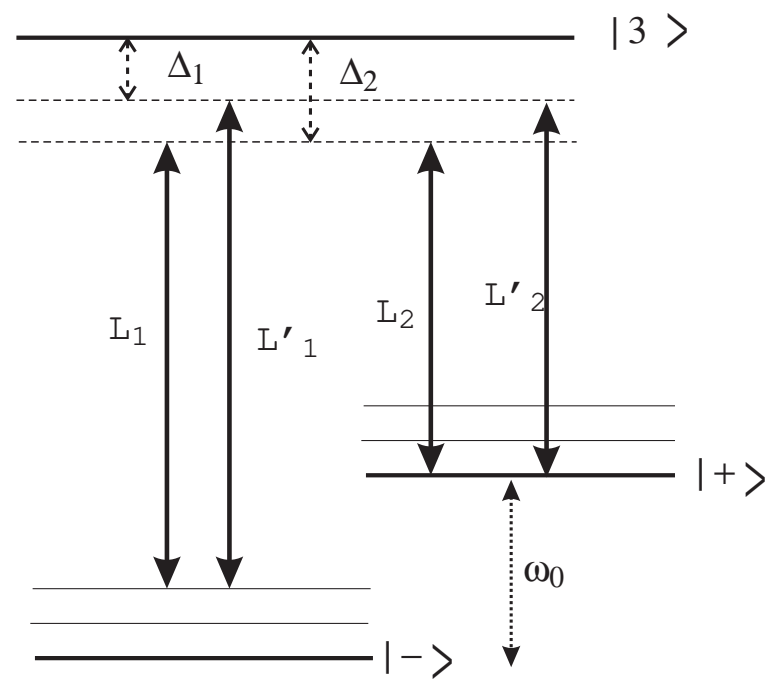

Figure 1. Three-level electronic system of the trapped ion and scheme of Raman couplings

vary $\eta_{R}$ by changing the Raman beams configurations. In this way it is possible to obtain very small values of the Lamb-Dicke parameter as required in our scheme.

For the scope of the paper it is important to underline that measurements of the electronic states are experimentally realizable with very high efficiency by means of the so called quantum jumps tecnhique [9, 10, 11]. The method essentially consists in coupling the ground electronic level $|-\rangle$ to a third auxiliary level $|r\rangle$ by means of another laser beam. The transition $|-\rangle \leftrightarrow|r\rangle$ is chosen to be a dipole allowed one. In this conditions, the presence of fluorescence detects the ion in the electronic ground level and its absence in the excited level. With this method state detection efficiency approaches $100 \%$, even if a modest number of scattered photons are detected.

\subsection{Dynamics}

Let's denote with $\left|n_{a}, n_{b}\right\rangle=\left|n_{a}\right\rangle\left|n_{b}\right\rangle$ the product of the vibrational Fock states along the $x$ and $y$ directions and suppose that the initial state of the ion has the form

$$
\begin{aligned}
|\Psi(0)\rangle=\left|\tau=1, j_{0}=\frac{N}{2}\right\rangle|-\rangle & \equiv \frac{1}{2^{N / 2}} \sum_{k=0}^{N}\left(\begin{array}{c}
N \\
k
\end{array}\right)^{1 / 2}|N-k, k\rangle|-\rangle \\
& \equiv \sum_{k=0}^{N} P_{k}|N-k, k\rangle|-\rangle
\end{aligned}
$$

The vibrational state $\left|\tau=1, j_{0}=\frac{N}{2}\right\rangle$ belongs to the class of the so called $\mathrm{SU}(2)$ coherent states defined as

$$
|\tau, j\rangle=\frac{1}{\left(1+|\tau|^{2}\right)^{j}} \sum_{k=0}^{2 j}\left(\begin{array}{c}
2 j \\
k
\end{array}\right)^{1 / 2} \tau^{k}|2 j-k, k\rangle
$$


where $\tau \in C, 2 j \in N$. The states $|N-k, k\rangle$ appearing in Eq. (8) are eigenstates of the operator $\left(\hat{a}^{\dagger} \hat{a}+\hat{b}^{\dagger} \hat{b}\right)$ all pertaining to the eigenvalue $N=2 j_{0}$ representing the initial total number of vibrational quanta. In particular, as pointed out by Gou and Knight [21], the $\mathrm{SU}(2)$ coherent state corresponding to the specific value $\tau=1,\left|\tau=1, j_{0}\right\rangle$ of a bidimensionally confined ion, coincides with the vibrational Fock state with $N=2 j_{0}$ quanta along the direction $\bar{x}$ forming an angle $\pi / 4$ relative to the $x$ axis. Since number states for the $1 \mathrm{D}$ motion of an ion in a rf-trap have been already experimentally realized (see [9, 11]), it is evident that the initial state of Eq. (8) could be easily prepared experimentally.

Then, if at $t=0$ the laser fields realizing the coupling between the $2 \mathrm{D}$ vibrational motion and the internal two-level system described by the Hamiltonian model of Eq. (7) is turned on, at any subsequent time instant $t$, the state of the system can be written as

$$
|\Psi(t)\rangle=\left|\varphi_{-}(t)\right\rangle|-\rangle+\left|\varphi_{+}(t)\right\rangle|+\rangle
$$

with

$$
\left|\varphi_{-}(t)\right\rangle=\sum_{k=0}^{N} P_{k} \cos \left(f_{k} t\right)|N-k, k\rangle
$$

and

$$
\left|\varphi_{+}(t)\right\rangle=-i \sum_{k=1}^{N-1} P_{k} \sin \left(f_{k} t\right)|N-k-1, k-1\rangle
$$

where

$$
f_{k}=2 g \sqrt{(N-k) k}
$$

are the Rabi frequencies. Eq. (10) shows that, starting from the factorized initial state $|\Psi(0)\rangle$ of Eq. (8), the Hamiltonian model (7) generates entanglement between the external and internal degrees of freedom of the trapped ion, giving rise to interesting dynamical consequences. In order to appreciate the meaning of this assertion, we focus our attention on the time evolution of the vibrational entropy

$$
S_{v}(t)=-\operatorname{Tr}\left[\rho_{v}(t) \ln \rho_{v}(t)\right]
$$

$\rho_{v}$ being the reduced density operator describing the $2 \mathrm{D}$ vibrational motion of the ion. A straightforward calculation gives

$$
S_{v}(t)=-\ln \left[c(t)^{c(t)}[1-c(t)]^{1-c(t)}\right]
$$

where

$$
c(t)=\sum_{k=0}^{N}\left|P_{k}\right|^{2} \cos ^{2}\left(f_{k} t\right)=\frac{1}{2}\left[1+\sum_{k=0}^{N}\left|P_{k}\right|^{2} \cos \left(2 f_{k} t\right)\right] .
$$

Exploiting an analytical method based on the analysis of the Rabi frequencies, it has been recently demonstrated in Ref. 22] that, starting from a total vibrational excitation number $N$, there exist an $N$-dependent time instant at which the internal and external degrees of freedom of the trapped ion are disentangled $(c(t)=1$ or $c(t)=0)$ or maximally 
(a)

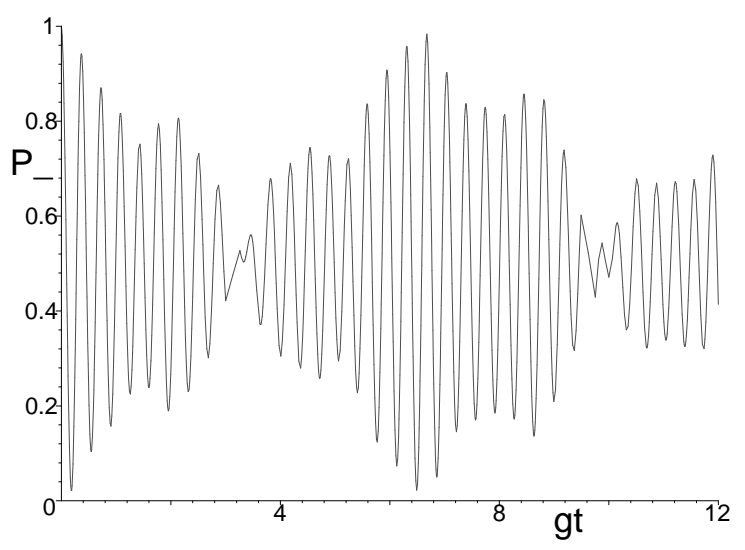

(b)

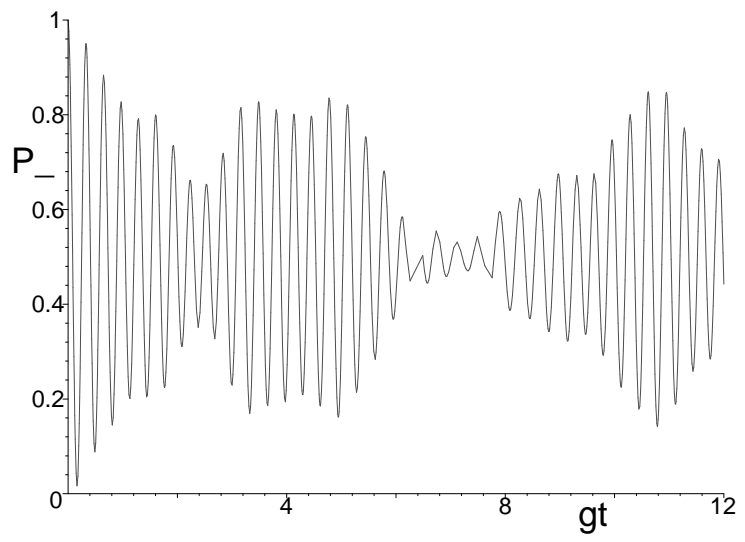

Figure 2. Time evolution of the probability to find the ion in the internal ground state $P_{-}(g t)$ for $N=9$ (a) and $N=10$ (b) respectively.

entangled $(c(t)=1 / 2)$. More in detail, it has been analytically proved that if $N \gg 1$ is odd, at the time instant $\bar{t}_{o}=\frac{\pi(N-1)}{4 g}$ one has $c\left(\bar{t}_{o}\right)=1\left(c\left(\bar{t}_{o}\right)=0\right)$ if $(N-1) / 2$ is even (odd). This implies that $S_{v}\left(\bar{t}_{o}\right)$ reaches its absolute minimum, indicating that the internal and external degrees of freedom manifest a marked tendency to disentangle each other. On the contrary, if $N \gg 1$ is even, at the time instant $\bar{t}_{e}=\frac{\pi N}{4 g}$, one has $c\left(\bar{t}_{e}\right)=1 / 2$ and therefore the vibrational entropy $S_{v}\left(\bar{t}_{e}\right)$ reaches its maximum value. This means that the vibrational and electronic degrees of freedom are, at this time instant, maximally entangled. Fig. 2 displays the time evolution of the probability $P_{-}(t) \equiv c(t)$ of finding the ion in its ground state (which can be measured with the technique described at the end of subsection 2.2) according to Eq. (16); Fig. 3 instead shows the vibrational entropy $S_{v}(t)$, as given by Eq. (15). In both figures, the cases of an initial total vibrational number $N=9$ (a) and $N=10$ (b) have been considered.

These figures illustrate in particular the existence of a $N$-dependent time instant at which the system under scrutiny exhibits different quantum behaviours dependent 
(a)

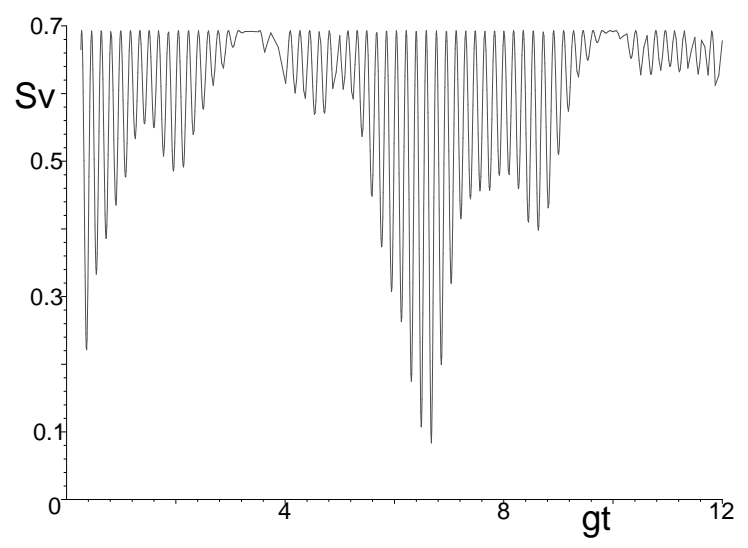

(b)

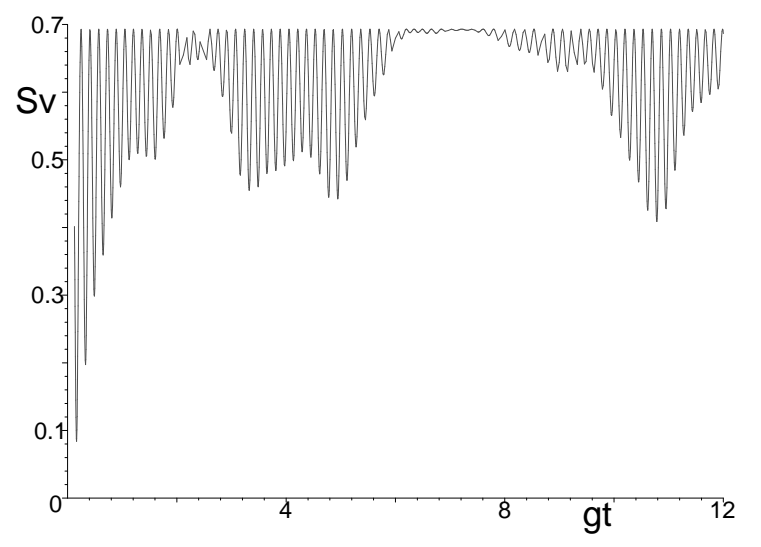

Figure 3. Time evolution of the vibrational entropy $S_{v}(g t)$ for $N=9$ (a) and $N=10$ (b) respectively.

on the parity of $N$. The interesting physical aspect is given by the peculiar nonclassical sensitivity to the granularity of the initial total number of vibrational quanta $N$. The physical origin of this intrinsically quantum behaviour stems from the specific twoboson coupling mechanism envisaged in this paper. In the rest of the paper we shall analyse the robustness of this quantum behaviour with respect to various experimental imperfections as the fluctuations of the intensity of the coupling lasers and a non-ideal initial state preparation

\section{Influence of non-dissipative decoherence sources}

In the previous section we have assumed perfect unitary evolution and initial state preparation for the system. This assumption is partially justified by the fact that a very good control of the quantum dynamics has been now achieved for trapped ions and also because they are well isolated from their environment. In fact, many experiments have 
shown that dissipative effects on the vibrational motion can be considered completely negligible for long intervals of time. Moreover, spontaneous emission is also practically irrelevant because the two internal states are two hyperfine levels of the ground state.

However, decoherence effects have been nonetheless observed in the motion of a laser driven trapped ion [9, 11]. In fact, even when the entanglement with the environment is negligible, fluctuations of some classical parameter of the system may cause nondissipative, phase-destroying effects. In the trapped ion case, phase decoherence is mainly caused by the fluctuations of the Rabi frequency, which are induced by the laser intensity fluctuations, making the coupling constant $g$ fluctuate. A quantitative explanation of the decoherence observed in the Rabi oscillations of Ref. [9], in terms of a fluctuating laser pulse area, has been recently provided by Bonifacio et al. in Ref. [37, using a model-independent formalism able to describe non-dissipative decoherence phenomena due to the fluctuations of classical parameters or internal variables of a system.

In the model-independent approach of Ref. [37], the dynamical quantities in the presence of non-dissipative decoherence can be obtained simply through an average over an appropriate probability distribution of the fluctuating parameter. In the trapped ion case, the random parameter is the positive dimensionless random variable $A(t)=\int_{0}^{t} d \xi g(\xi)$, which is proportional to the laser pulse area. In fact, the quantity $g(\xi)$ is a stochastic coupling constant, whose fluctuations have to be traced back to the laser intensity fluctuations. The time evolution of a generic dynamical quantity $O(t)$ becomes therefore the averaged quantity

$$
\bar{O}(t)=\int_{0}^{\infty} d A P(t, A) O(A) .
$$

The probability distribution $P(t, A)$ is the Gamma distribution function

$$
P(t, A)=\frac{e^{-A / g \tau}}{g \tau} \frac{(A / g \tau)^{(t / \tau)-1}}{\Gamma(t / \tau)},
$$

obtained in Ref. 37 by imposing the semigroup condition for the averaged density matrix of the system. The physical meaning of the parameters $g$ and $\tau$ in Eq. (18) can be easily understood by considering the mean and the variance of the probability distribution (18),

$$
\begin{aligned}
& \langle A\rangle=g t \\
& \sigma^{2}(A)=\left\langle A^{2}\right\rangle-\langle A\rangle^{2}=g^{2} t \tau,
\end{aligned}
$$

implying that $g$ has now to be meant as a mean coupling constant, and that $\tau$ quantifies the strength of $A$ fluctuations. In Ref. [37] the decay of the Rabi oscillations observed in Ref. [9] is well fitted assuming $\tau \simeq 1.5 \cdot 10^{-8} \mathrm{sec}$ (see also 44]). In this case, the relevant experimental timescale $t$ is much larger than $\tau$ and in this limit the Gamma distribution function can be well approximated by the following Gaussian distribution

$$
P(t, A) \simeq \frac{1}{\sqrt{2 \pi g^{2} t \tau}} \exp \left\{-\frac{(A-g t)^{2}}{2 g^{2} t \tau}\right\} .
$$




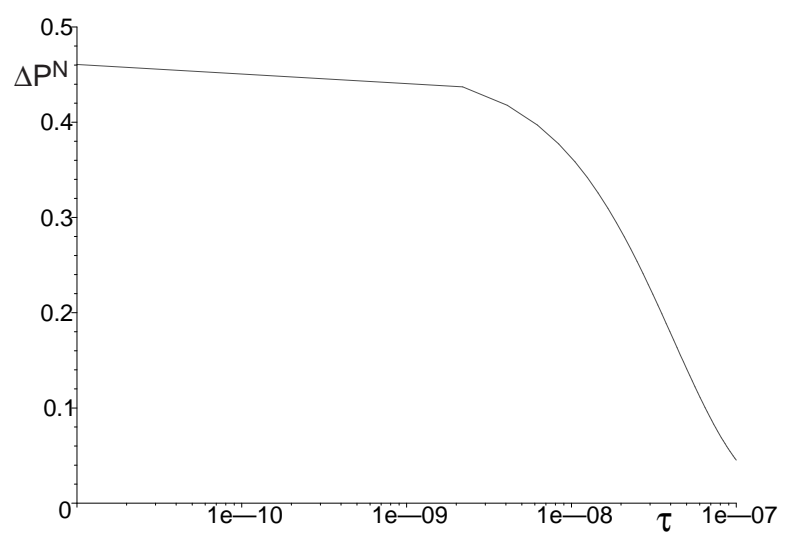

Figure 4. The probability difference $\Delta P^{N}$ defined in the text, at the time instant $\bar{t}$, as a function of the laser pulse area fluctuation strength parameter $\tau$ (expressed in seconds), in the case of a total initial vibrational quanta $N=9$ and for $g=10^{5} \mathrm{~Hz}$.

The experimentally observed quantity is the probability of finding the ion in its ground electronic state $P_{-}(t) \equiv c(t)$. In the presence of the fluctuating coupling constant $g(\xi)$ caused by the intensity fluctuations of the coupling lasers, this probability has therefore to be averaged over the Gaussian distribution (21). Using Eq. (16) we find

$$
\bar{P}_{-}(g t)=\frac{1}{2}\left[1+\sum_{k=0}^{N} e^{-8 g^{2} \tau(N-k) k t} \cos (4 \sqrt{(N-k) k} g t)\right],
$$

where the quantum coherent oscillations at frequencies $f_{k}$ are now exponentially decaying because of the non-dissipative decoherence.

To quantify the robustness of the parity-dependent entanglement with respect to the various experimental imperfections, we have considered the quantity $\Delta P^{N}$, defined as the difference between the values assumed by $\bar{P}_{-}(g \bar{t})$ in correspondence to $N$ (odd) and $N+1$ total initial vibrational excitations, at the "intermediate" time $\bar{t}=\frac{\bar{t}_{e}+\bar{t}_{o}}{2}$. With no imperfections and $N \gg 1$, one has $\bar{t} \sim \bar{t}_{e} \sim \bar{t}_{o}$, and $\bar{P}_{-}^{N=o d d}(g \bar{t}) \sim 1, \bar{P}_{-}^{N+1}(g \bar{t}) \sim 1 / 2$, so that $\Delta P^{N} \simeq 1 / 2$ whatever $N$ is. We emphasize that such a result highlights the fact that the probability $P_{-}(\bar{t})$ of finding the ion in its electronic ground level at $t=\bar{t}$ depends on $N$ being even or odd. As long as the effect of phase decoherence increases, the difference between the odd $N$ case and the even $N$ case tends to zero, because in both cases the system state becomes the same statistical mixture and $\Delta P^{N}$ tends to zero.

In Fig. 4 we show the dependence of the probability difference $\Delta P^{N}$ as a function of the laser pulse area fluctuation strength $\tau$, in the case when $N=9$ and $g=10^{5} \mathrm{~Hz}$. One can see the expected decay to zero of $\Delta P^{N}$ for increasing $\tau$ (i.e., increasing laser intensity fluctuations); in particular one has a well visible transition from a quantum behaviour at small $\tau$ to a completely decoherent behaviour at larger $\tau$, taking place at a threshold value $\tau_{t h} \sim 3 \cdot 10^{-8}$ sec. Since current experimental situations correspond to $\tau \leq 10^{-8}$ sec, this means that the parity-dependent entanglement effect described in the 
preceding section can be experimentally detected even with the unavoidable presence of non-dissipative decoherence sources, as for example laser intensity fluctuations.

\section{Imprecision in the initial state preparation}

Another important condition for the observability of the parity effect reported in this paper is the possibility of preparing the initial vibrational Fock state $|N\rangle$ along the direction $X^{\prime}$ at $\pi / 4$ radians with respect to $X$. It is however not difficult to guess that at the end of any scheme aimed at preparing a vibrational ionic Fock state containing exactly $N$ vibrational quanta, the state effectively reached is a vibrational density matrix $\rho_{v}(0)$ containing also diagonal terms diffeent from $|N\rangle\langle N|$ in the total vibrational energy eigenbasis. Of course, such a mixture of initial odd and even total vibrational quanta counters the possibility of observing the parity dependent entanglement. However, we shall show that the degree of preparation efficiency required in order to keep the parity effect in the dynamics of our system appears realistically in the grasp of the experimentalists. In other words we shall prove that the occurrence of parity effects, although attenuated, turns out to be compatible with the initial state preparation fidelity presently achievable for ionic vibrational states.

We assume that at $t=0$ the initial vibrational state can be described as follows:

$$
\rho_{v}(0)=\sum_{m=0}^{\infty} \mathcal{N} e^{-(m-N)^{2} / 2 \Delta^{2}}|m, 0\rangle\langle m, 0|,
$$

$\mathcal{N}$ being the normalization constant. Equation (23) is a gaussian weighted mixture of pure Fock states $|m, 0\rangle\langle m, 0|$ along the direction $X^{\prime}$, centered on a prefixed number $N$ of vibrational quanta and controlled by a width parameter $\Delta$. It is immediate to see that when $\Delta \rightarrow 0 \rho_{v}(0)$ tends to $|N, 0\rangle\langle N, 0|$. The form given to $\rho_{v}(0)$ suggests the introduction of the parameter

$$
\eta=1-\frac{p_{N+1}}{p_{N}}=1-e^{-1 / 2 \Delta^{2}}
$$

describing the efficiency of initial state preparation. $p_{N+1}$ and $p_{N}$ appearing in Eq. (24) are the probabilities of having $N+1$ or $N$ quanta in the initial vibrational Fock state. With the help of equations (22) and (23), it is not difficult to verify that, in the presence of both laser intensity fluctuations and imperfect initial state preparation, the probability to find the ion in the internal ground state $|-\rangle, \bar{P}_{-}(g t)$, assumes the form

$$
\bar{P}_{-}(g t)=\frac{1}{2}\left[1+\sum_{m=0}^{\infty} \mathcal{N} e^{-(m-N)^{2} / 2 \Delta^{2}} \sum_{k=0}^{m} e^{-8 g^{2} \tau(m-k) k t} \cos (4 \sqrt{(m-k) k} g t)\right]
$$

As we have done in the preceding section, we quantify the robustness of the paritydependent effect in terms of the probability difference $\Delta P^{N}$ defined above. In Fig. 5 $\Delta P^{N}$ is plotted as a function of the preparation efficiency $\eta$ for three different values of the fluctuation strength parameter, $\tau=10^{-9} \sec$ (solid line), $\tau=10^{-8}$ sec (dotted line) and $\tau=10^{-7}$ sec (dashed line), again in the case $N=9, g=10^{5} \mathrm{~Hz}$. As expected, one has a steady increase of $\Delta P^{N}$ for increasing preparation efficiency. In particular, if we 


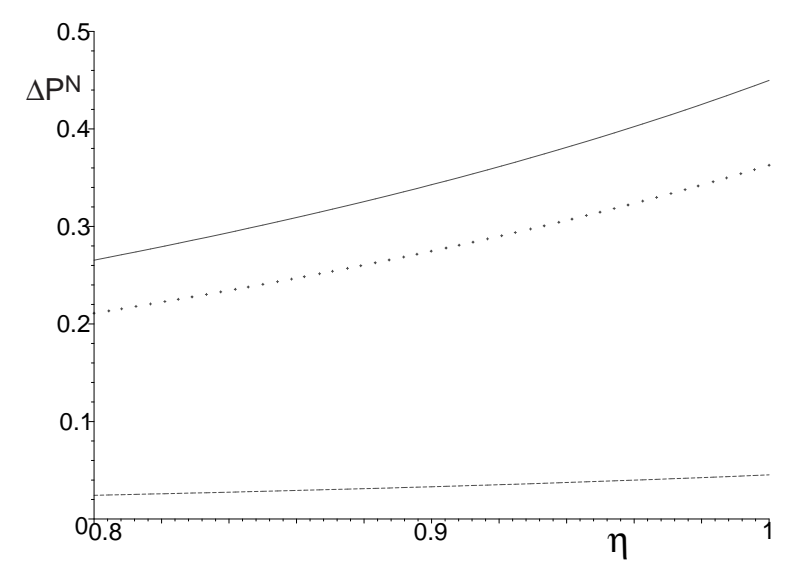

Figure 5. The probability difference $\Delta P^{N}$ at the time instant $\bar{t}$ as a function of the efficiency of the initial state preparation $\eta$ (see Eq. (24)) for $\tau=10^{-9} \sec$ (solid line), $\tau=10^{-8} \sec$ (dotted line) and $\tau=10^{-7} \sec$ (dashed line). The other parameters are as in Fig. 3.

assume the realistic parameters $\eta=0.9$ and $\tau=10^{-8} \mathrm{sec}$, one can see that the parity dependent entanglement effect is still visible, although attenuated by almost $40 \%$.

\section{Conclusions}

In this paper we have analyzed how some experimental imprecisions influence a nonclassical effect we have brought to light and discussed in a previous paper [22], namely the parity dependent entanglement effect. According to this effect by changing only one vibrational quantum in an initial condition characterized by a total number of oscillatory quanta $N \gg 1$, the state of the system, at approximately the same instant of time $t_{e} \simeq t_{o}$, drastically changes. Indeed at this time instant the vibrational and electronic degrees of freedom of the ion are maximally entangled (i.e. maximum reduced entropy) for even $N$ and disentangled for odd $N$.

The parity dependent quantum effect has a nonclassical origin since it stems from the granularity of the oscillatory energy of the center of mass of the ion. For this reason we believe it's worth being experimentally verified. We have thus studied the robustness of the effect with respect to various experimental imperfection. Since single trapped ions are essentially dissipationless systems, we have considered the effect of non-dissipative decoherence sources as the intensity fluctuations of the coupling lasers, and also the effect of an imperfect initial state preparation. The main result of the paper is that the parity-dependent entanglement is quite robust against these experimental imperfections and that it can be experimentally seen using presently available technology. 


\section{Acknowledgements}

The authors of Palermo University acknowledge financial support from CRRNSMRegione Sicilia. One of the authors (A.N.) is indebted to MURST and FSE for supporting this work through Assegno di Ricerca.

\section{References}

[1] W.M. Itano and D.J. Wineland: Phys. Rev. A 25 (1982) 35

[2] D.J. Wineland, W.M. Itano, J.C. Bergquist and R.G. Hulet: Phys. Rev. A 36 (1987) 2220

[3] F. Diedrich, J.C. Bergquist, W.M. Itano and D.J. Wineland: Phys. Rev. Lett. 62 (1989) 403

[4] C. Monroe, D.M. Meekhof, B.E. King, J.R. Jefferts, W.M. Itano and D. J. Wineland: Phys. Rev. Lett. 75 (1995) 4011

[5] Y. Castin and J. Dalibard: Europhys. Lett. 14 (1991) 761

[6] C.I. Westbrook, R.N. Watts, C.E. Tanner, S.L. Rolston, W.D. Phillips, P.D. Lett and P.L. Gould:Phys. Rev. Lett. 65 (1990) 33

[7] M. Kasevich and S. Chu: Phys. Rev. Lett. 69 (1992) 1741

[8] C. Monroe, D.M. Meekhof, B.E. King, W.M. Itano and D. J. Wineland: Phys. Rev. Lett. 75 (1995) 4714

[9] D.M. Meekhof, C. Monroe, B.E. King, W.M. Itano and D. J. Wineland: Phys. Rev. Lett. 76 (1996) 1796

[10] C. Monroe, D.M. Meekhof, B.E. King and D.J. Wineland: Science 212, (1996) 1131

[11] Ch. Roos, Th. Zeiger, H. Rohde, H.C. Nägerl, J. Eschner, D. Leibfried, F. Schmidt-Kaler, R. Blatt: Phys. Rev. Lett. 83 (1999) 4713

[12] F. Schmidt-Kaler, Ch. Roos, H.C. Nägerl et al. J. Mod. Opt. 47 (2000) 2573

[13] J.I. Cirac, R. Blatt, A.S. Parkins and P. Zoller Phys. Rev. Lett. 70 (1993) 762

[14] J.I. Cirac, R. Blatt and P. Zoller: Phys. Rev. A 49 (1994) R3174

[15] R. Blatt, J.I. Cirac, A.S. Parkins and P. Zoller: Phys. Rev. A 52 (1985) 518

[16] J.I. Cirac, A.S. Parkins, R. Blatt and P. Zoller: Phys. Rev. Lett. 70 (1993) 556

[17] H. Zeng and F. Lin: Phys. Rev. A 52 (1995) 809

[18] C.C. Gerry, S.-C. Gou, and J. Steinbach: Phys. Rev A 55 (1997) 630

[19] S.-C. Gou, J. Steinbach and P.L. Knight, Phys. Rev A 54 (1996) 4315

[20] S.-C. Gou, J. Steinbach and P.L. Knight Phys. Rev A 54 (1996) R1014

[21] S.-C. Gou and P.L. Knight Phys. Rev A 54 (1996) 1682

[22] S. Maniscalco, A. Messina and A. Napoli Phys. Rev A 61 (2000) 053806

[23] S. Maniscalco, A. Messina and A. Napoli J. Mod. Opt. 47 (2000) 2113

[24] J.I. Cirac and P. Zoller: Phys. Rev. Lett. 74 (1995) 4091

[25] L. Li and G. Guo: Phys. Rev. A 60 (1999) 696

[26] D. Leibfried, D.M. Meekhof, B.E. King, C. Monroe, W. M. Itano, and D. J. Wineland: Phys. Rev. Lett. 77 (1996) 4281

[27] S. Wallentowitz and W. Vogel: Phys. Rev. Lett. 75 (1995) 2932

[28] J.F. Poyatos, R. Walser, J.I. Cirac and P.Zoller: Phys. Rev. A 53 (1996) R1966

[29] P.J. Bardroff, C. Leichtle, G. Schrade, and W. P. Schleich: Phys. Rev. Lett. 77 (1996) 2198

[30] S. Wallentowitz and W. Vogel: Phys. Rev. A 55 (1997) 4438

[31] R. Huesmann, Ch. Balzer, Ph. Courteille, W. Neuhauser and P.E. Toschek: Phys. Rev. Lett. 82 (1999) 1611

[32] S. Wallentowitz and W. Vogel: Phys. Rev. A 58 (1998) 679

[33] S. Wallentowitz and W. Vogel: Phys. Rev. A 59 (1999) 531

[34] R.L. de Matos Filho and W. Vogel: Phys. Rev. A 58 (1998) R1661

[35] J. Steinbach, J. Twamley and P.L. Knight: Phys. Rev. A 56 (1997) R1661 
[36] D.J. Wineland, C. Monroe, W.M. Itano, D. Leibfried, B.F. King and D.M. Meekhof, J. Res. Natl. Inst. Stand. Technol. 103 (1998) 259

[37] R. Bonifacio, S. Olivares, P. Tombesi, D. Vitali, Phys. Rev. A 60 (2000) 053802

[38] K. Wódkiewicz and J.H. Eberly: J. Opt. Soc. Am. B 3 (1985) 458

[39] V. Bužek and T. Quang: J. Opt. Soc. Am. B 6 (1989) 2447

[40] S.A. Gardiner, J.I. Cirac, and P.Zoller: Phys. Rev. A 55 (1997) 1683

[41] C. D'Helon and G.J. Milburn: Phys. Rev. A 54 (1996) R25

[42] L. Davidovich, M. Orszag and N. Zagury: Phys. Rev. A 54 (1996) 5118

[43] R.L. de Matos Filho and W. Vogel: Phys. Rev. Lett. 76 (1996) 4520

[44] S. Schneider and G.J. Milburn, Phys. Rev. A 573748 (1998) 